\title{
Increased thrombin generation in splanchnic vein thrombosis is related to the presence of liver cirrhosis and not to the thrombotic event
}

\author{
Roza Chaireti, Rupesh Rajani, Annika Bergquist, Tor Melin, Inga-Lill Friis-Liby, Marjo \\ Kapraali, Stergios Kechagias, Tomas L. Lindahl and Sven Almer
}

\section{Linköping University Post Print}

\section{Tweet}

N.B.: When citing this work, cite the original article.

Original Publication:

Roza Chaireti, Rupesh Rajani, Annika Bergquist, Tor Melin, Inga-Lill Friis-Liby, Marjo Kapraali, Stergios Kechagias, Tomas L. Lindahl and Sven Almer, Increased thrombin generation in splanchnic vein thrombosis is related to the presence of liver cirrhosis and not to the thrombotic event, 2014, Thrombosis Research, (134), 2, 455-461.

http://dx.doi.org/10.1016/j.thromres.2014.05.012

Copyright: Elsevier

http://www.elsevier.com/

Postprint available at: Linköping University Electronic Press http://urn.kb.se/resolve?urn=urn:nbn:se:liu:diva-100216 
Increased thrombin generation in splanchnic vein thrombosis is related to the presence of liver cirrhosis and not to the thrombotic event.

Roza Chaireti ${ }^{1,2^{*}}$, Rupesh Rajani ${ }^{3^{*}}$, Annika Bergquist ${ }^{3}$, Tor Melin ${ }^{4}$, Inga-Lill Friis-Liby ${ }^{5}$, Marjo Kapraali ${ }^{6}$, Stergios Kechagias ${ }^{7,8}$, Tomas L. Lindahl ${ }^{1}$ and Sven Almer $r^{9,10}$

1Department of Clinical and Experimental Medicine, Linköping University, Linköping,

Sweden

${ }^{2}$ Department of Haematology, Karolinska University Hospital, Stockholm, Sweden ${ }^{3}$ Division of Gastroenterology \& Hepatology, Karolinska Institutet, Karolinska University Hospital, Stockholm, Sweden

${ }^{4}$ Division of Gastroenterology \& Hepatology, University Hospital, Lund, Sweden ${ }^{5}$ Department of Medicine, Sahlgrenska University Hospital, Gothenburg, Sweden ${ }^{6}$ Karolinska Institutet, Department of Clinical Sciences Danderyd Hospital, Division of Medicine, Stockholm, Sweden

7Department of Gastroenterology and Hepatology, Linköping University Hospital, Linköping, Sweden

${ }^{8}$ Department of Medical and Health Sciences, Linköping University, Linköping,

Sweden

${ }^{9}$ Karolinska Institutet, Department of Medicine, Solna, Sweden

${ }^{10}$ GastroCentrum, Karolinska University hospital, Stockholm, Sweden

${ }^{*}$ Equal contribution

\section{Correspondence:}


Roza Chaireti

Department of Haematology

Karolinska University Hospital

Stockholm, Sweden

Telephone: +468517 70000

e-mail: roza.chaireti@karolinska.se

Word count: $\quad 4802$ (Main body)

250 (Abstract)

Abbreviations: CLD: chronic liver disease, CP: Child Pugh, PT-INR: prothrombin time-international normalized ratio, VTE: venous thromboembolism, RR: relative risk, ETP: endogenous thrombin potential, PC: protein C, FVIII: factor VIII, BCS: BuddChiari syndrome, NC-PVT: non-cirrhotic portal vein thrombosis, C-PVT: cirrhotic portal vein thrombosis, AF: atrial fibrillation, PPP: platelet poor plasma, CAT: calibrated automated assay, $\mathrm{nM}$ : nanomolar, ttpeak: time to peak, IU/mL: international units/milliliter, hsCRP: high sensitivity CRP, TM: thrombomodulin 


\section{Abstract}

Introduction: In recent years there have been increasing evidence associating liver disease with hypercoagulability, rather than bleeding. The aim of the study was to evaluate the haemostatic potential in patients with liver disease.

Patients and methods: We measured thrombin generation in the presence and absence of thrombomodulin in patients with portal vein thrombosis (PVT, $n=47$ ), Budd-Chiari syndrome (BCS, $n=15)$ and cirrhosis $(n=24)$ and compared the results to those obtained from healthy controls $(n=21)$. Fifteen patients with PVT and ten patients with BCS were treated with warfarin and were compared to an equal number of patients with atrial fibrillation matched for Prothrombin time-International Normalized Ratio. We assessed resistance to thrombomodulin by using ratios [marker measured in the presence/absence of thrombomodulin].

Results: There were no differences in thrombin generation between patients on warfarin treatment and their controls. Cirrhotic patients generated more thrombin in the presence of thrombomodulin and exhibited thrombomodulin resistance compared to controls $[p=0.006$ for endogenous thrombin potential $(E T P)$ and $p<0.001$ for peak thrombin and both ratios ETP and peak] and patients with non-cirrhotic PVT $(p=0.001, p=0.006, p<0.001, p<0.001$ for ETP, peak, ratio ETP, ratio peak, respectively). The patients with cirrhotic PVT exhibited higher ETP $(p=0.044)$ and 
peak $(p=0.02)$ in the presence of thrombomodulin than controls, as well as thrombomodulin resistance (ETP and peak ratios: $p=0.001$ ).

Conclusions: Hypercoagulability and thrombomodulin resistance in patients with cirrhosis were independent of the presence of splanchnic vein thrombosis. The hypercoagulability in patients with cirrhotic PVT could have implications for considering longer or more intensive treatment with anticoagulants in this group.

Keywords: thrombin generation, portal vein thrombosis, Budd-Chiari syndrome, cirrhosis, thrombomodulin 


\section{Introduction}

The liver plays a central role in maintaining the haemostatic balance by synthesizing several procoagulant, anticoagulant and fibrinolytic proteins [1]. Impaired liver function, as in chronic liver disease (CLD), results in thrombocytopenia, low levels of many procoagulant and anticoagulant factors and changes in fibrinolysis [2-5] and is reflected by the Child-Pugh (CP) class [6, 7].

CLD has previously been considered a prototype for bleeding disorders, mainly due to abnormal values of coagulation tests such as prothrombin time-International Normalized Ratio (PT-INR) and activated partial thromboplastin time [2, 7]. However, abnormal coagulation tests do not necessarily correspond to or predict the risk of bleeding manifestations or even the degree of coagulopathy, as they provide inadequate information on the complex mechanisms that regulate the coagulation cascade [8]. It is thus desirable to find new haemostatic tests that better estimate the degree of coagulation imbalance in patients with CLD.

The dominance of bleeding complications in CLD has been challenged [9-11]. More recent data [10] indicate that hypercoagulability might be frequently associated with CLD and cirrhosis, meaning that these patients are not 'naturally anticoagulated'; on the contrary they have an increased risk for thromboembolic complications. In a large, nationwide, case-control, Danish study patients with liver disease had an increased relative risk (RR) of VTE, both in cirrhotic (RR 1.74) and non-cirrhotic liver disease (RR 1.87) [12]. Furthermore, VTE is the main reason behind $1 \%-1.8 \%$ of hospital admissions for patients with cirrhosis $[13,14]$. 
The complex resulting from the binding of the protein thrombomodulin to thrombin activates the natural anticoagulant protein $\mathrm{C}(\mathrm{PC})$. Activated PC binds to its co-factor protein $S$ and subsequently inhibits the activated forms of the procoagulants factor VIII (FVIII) and factor V, thus inhibiting thrombin generation. Thrombomodulin inhibits thrombin generation even in vitro, which is evident by the lower results of thrombin generation assays when thrombomodulin is added to the plasma sample [2]. Thrombin generation assays are coagulation tests that provide a global measure of haemostatic potential [15]. The effect of thrombomodulin, i.e. inhibiting thrombin generation, is more evident in plasma from healthy individuals than in plasma from patients with CLD, indicating partial resistance to thrombomodulin [7]. Resistance to thrombomodulin as expressed by the ratio [thrombin generation marker measured in the presence/in the absence of thrombomodulin] is a marker for hypercoagulability in vitro and has been described in patients with liver disease as a result of high levels of FVIII and low levels of PC in those patients [2]. Tripodi et al showed that thrombin generation in cirrhotic patients, assessed as endogenous thrombin potential (ETP) in the presence of thrombomodulin was nearly identical to thrombin generation in controls, in contrast to the belief that patients with CLD are at increased bleeding risk [9]. Thrombin generation assays provide therefore a more accurate picture of the haemostatic potential in patients with hepatic disease, in contrast to traditional coagulation tests.

The Budd-Chiari syndrome (BCS) is a rare condition characterized by obstruction of the hepatic venous outflow tract mainly due to thrombosis of the hepatic veins [16]. 
The etiology of BCS has been associated with myeloproliferative diseases [17] and hereditary thrombophilic conditions, such as the presence of factor V Leiden [18].

Non-cirrhotic portal vein thrombosis (NC-PVT) is the main cause of presinusoidal portal hypertension in adults and children in the Western world [19]. NC-PVT can either be idiopathic, mediated by inherited or acquired prothrombotic conditions or associated with local conditions, for example inflammation and abdominal surgery. NC-PVT is often caused by a combination of local and systemic factors [20, 21]. Cirrhotic-PVT (C-PVT) is a common complication during the course of cirrhosis, occurring mainly during the advanced stages of the disease [22].

In this report, we study thrombin generation, as well as the resistance to thrombomodulin in patients with cirrhosis, C-PVT, NC-PVT, BCS and in controls in order to investigate the haemostatic potential in vitro in patients with various forms of CLD. The ratio [thrombin generation marker measured in the presence/in the absence of thrombomodulin] is a marker of hypercoagulability in vitro as it expresses the grade of increased FVIII and decreased PC in the plasma of patients with CLD [9]. The hypothesis behind this study is that findings supporting hypercoagulability in vitro (high thrombin generation and thrombomodulin resistance) could be linked not only to cirrhosis but even to in vivo hypercoagulability in these patients, in this case the presence of splanchnic thrombosis.

\section{Patients and Methods}

\section{Patients}


This study was performed in collaboration with the Swedish Internal Medicine Liver Club, SILK, a nationwide network of university hepatologists. Forty-seven PVT patients (thirty-six with NC-PVT and eleven with C-PVT) diagnosed 1995-2009, fifteen patients with BCS diagnosed 1988-2009 and twenty-four patients with cirrhosis diagnosed 2002-2010 were included. The basic characteristics of the patients are shown in table 1.

The causes of cirrhosis in patients with C-PVT were: hepatitis C virus $(n=4)$, alcoholic liver disease $(n=2)$, cryptogenic $(n=2)$, autoimmune hepatitis $(n=2)$, hepatitis $B$ virus $(n=1)$ and for the patients with cirrhosis and no PVT: alcoholic liver disease $(n=10)$, non-alcoholic fatty liver disease $(n=4)$, hepatitis $C$ virus $(n=4)$, autoimmune hepatitis $(n=3)$, hemochromatosis $(n=1)$, cryptogenic $(n=1)$ and primary sclerosing cholangitis $(n=1)$.

\section{Controls for thrombin generation measurement}

We measured thrombin generation in twenty-one healthy volunteers [eight male and thirteen female, median age (range): 57 years (29-69)]. The term 'control group' in the text refers to this group, unless otherwise specified.

Fifteen patients with PVT (NC-PVT $n=13$, C-PVT $n=2$ ) and ten patients with BCS were under treatment with warfarin at the time of the blood sampling. We therefore investigated an equal number of otherwise healthy patients with atrial fibrillation (AF) on warfarin as prophylaxis against stroke. These AF patients were age-, gender- and 
PT-INR matched to the PVT patients treated with warfarin. The AF patients who served as controls for the BCS patients were gender- and PT-INR -matched, as their median age was very young (32 years) and most of the patients in the AF cohort were older. The median (range) PT-INR (both patients and controls) was 2.9 (1.93.5).

\section{Blood sampling and handling}

The median time between diagnosis and sampling was 46 months (1-164) for PVT, 78 months (1-246) for BCS and 13 months (0-96) for cirrhotic patients. Blood samples were collected after an overnight fast from antecubital veins in sodium 1/10 volume of $0.13 \mathrm{mmol} / \mathrm{L}$ citrate, EDTA and serum tubes (Becton Dickinson, Meylan, France). The samples were centrifuged within two hours at $2500 \mathrm{~g}$ for 10 minutes at room temperature and stored at $-70^{\circ} \mathrm{C}$ pending analyses.

The citrate samples used for analysis of thrombin generation were centrifuged once more at $2500 \mathrm{~g}$ for 10 minutes at room temperature following thawing.

Thrombin generation measurement by the Calibrated Automated Thrombogram

Thrombin generation was measured by the Calibrated Automated Thrombogram $\left(\mathrm{CAT}^{\circledR}\right)$ as described in the Thrombogram Guide by Thrombinoscope BV (Maastricht, the Netherlands) [23]. 
The samples used were platelet poor plasma (PPP) obtained from whole blood as described above. The frozen plasma was thawed by immersion into a water bath at $37^{\circ} \mathrm{C}$ immediately prior to analysis. Each sample was assessed with and without thrombomodulin in duplicate. All thrombin generation analyses were performed by the first author in order to avoid variability in the performance of the experiments.

Briefly, each experiment used two sets of readings, one from a well in which thrombin generation takes place (thrombin generation well) and a second one from a well to which a calibrator has been added. To each well, 80 microliter $(\mu l)$ of plasma was added. The thrombin generation wells received $20 \mu \mathrm{l}$ of buffer, containing the trigger but no $\mathrm{Ca}^{2+}$, and the calibrator wells $20 \mu \mathrm{l}$ of the a2macroglobulin-thrombin complex solution. Soluble rabbit lung thrombomodulin (American Diagnostica Inc, Stamford, CT) was added at a final concentration of 4 nanomolar/L, nM/L, as described previously by others $[9,20]$. The mixture of PPP reagent (trigger) and PPP used in the assay contained 5 picomolar tissue factor and 4 micromolar phospholipids. The plate was then placed in the fluorometer. The instrument dispensed $20 \mu \mathrm{l}$ of fluorogenic substrate and buffer (FluCa) to each well that was to be measured. During the measurement, the program compared the readings from the thrombin generation and the calibrator wells, calculating thrombin generation.

The markers measured were: lagtime (clotting time, the moment at which thrombin generation begins), ETP (the total amount of thrombin generated, in nanomolar, thrombin*minute), Peak Height (maximal thrombin concentration, in nM thrombin) and ttPeak (time to peak height, i.e. maximal thrombin concentration, in minutes). 


\section{Measurement of FVIII, antithrombin and high sensitivity CRP}

We measured FVIII and antithrombin as these coagulation parameters are representative of pro- and anticoagulant activity and are not affected by treatment with warfarin. FVIII was measured by a chromogenic method using commercial kits and reagents from Instrumentation Laboratory (IL ${ }^{\mathrm{TM}}$, Milan, Italy). Antithrombin was measured by a chromogenic method from the same manufacturer. The results for FVIII and antithrombin are given as international units/milliliter $(\mathrm{IU} / \mathrm{mL})$. All measurements were performed on the ACL TOP instrument, Instrumentation Laboratory.

The samples for FVIII analysis were collected upon a follow-up visit and not at the time of the acute event in order to avoid increased values due to inflammation.

We measured high-sensitivity C-reactive protein (hs-CRP) as a marker for inflammation. hs-CRP was measured in all patients by means of the clinical chemistry automated analyser Advia 1800 (Siemens Healthcare Diagnostics, Stockholm). The results are given as milligram/liter (mg/L).

\section{Statistical analysis}

Continuous variables were expressed as medians and ranges and tested for statistical significance with the nonparametric Mann-Whitney $U$ test. The least squares multiple regression analysis was used for evaluating the associations between markers. A two-sided $P$ value of .05 or less was considered statistically 
significant. Statistical analyses were performed with the SPSS software package, version 17.0 (SPSS, Inc, Chicago, IL).

\section{Results}

The median (range) for thrombin generation markers for the patients and controls without warfarin treatment are presented in table 2 and for the patients and controls on warfarin treatment in tables 3 and 4 . All values for thrombin generation markers and ratios are presented as median (range), unless otherwise specified.

The results for thrombin generation presented below refer to the measurements in the presence of thrombomodulin, unless otherwise specified.

The term "ratio" refers to the ratio of [thrombin generation marker measured in the presence/in the absence of thrombomodulin] unless otherwise specified.

\section{Thrombin generation and thrombomodulin resistance for all patients with PVT without warfarin}

No differences were found for thrombin generation markers in the presence of thrombomodulin for patients with PVT without warfarin treatment compared to healthy controls. The ratios for ETP and peak were significantly higher for the patients with PVT compared to controls ( ETP ratio: $p=0.02$, peak ratio: $p=0.009$ ). 
No significant associations were found between thrombin generation markers, FVIII, antithrombin and hsCRP in this group (data not shown).

\section{Thrombin generation and thrombomodulin resistance for patients with C- PVT and NC-PVT without warfarin}

ETP and peak in patients with C-PVT were higher than in patients with NC-PVT [ETP: $p=0.068$, non-significant, $882(406-1274) n M^{*} \min$ vs. 432 (275-1689) nMmin. Peak: $\mathrm{p}=0.037,166(102-206) \mathrm{nM}$ vs. 79 (35-257) nM] (figures 1,2). The ratios for lagtime, ETP and peak were also higher in the C-PVT group compared to the group with NC-PVT (lagtime: $p=0.007$, ETP: $p=0.018$, peak: $p=0.033$ ).

Neither thrombin generation markers in the presence of thrombomodulin nor ratios differed significantly between patients with NC-PVT and controls.

However, ETP and peak were significantly higher in the group with C-PVT compared to controls [ETP: $p=0.044,882(406-1274) n M^{*} \min$ vs. $593(296-1406) n M^{*} \min$, peak: $\mathrm{p}=0.020,166(102-206) \mathrm{nM}$ vs 117 (56-318) nM] (figures 1, 2). The ratios for ETP and peak were also higher in the C-PVT group compared to controls (ratio ETP and peak: $p=0.001$ )

Neither the thrombin generation markers nor the ratios were significantly different between CP classes. ETP (in the presence of thrombomodulin) was associated with the CP class, however this did not reach statistical significance $(p=0.106)$. 


\section{Thrombin generation and thrombomodulin resistance in patients with Budd-} Chiari syndrome without warfarin

None of the thrombin generation markers were significantly different between BCS patients and their controls.

The ratios for lagtime, ETP and peak were higher in the BCS group compared to the ratios for the controls (lagtime: $p=0.012$, ETP: $p=0.049$, peak: $p=0.077$, nonsignificant).

No significant associations were found between thrombin generation markers, FVIII, AT and hsCRP in this group.

\section{Thrombin generation and thrombomodulin resistance in patients with cirrhosis and no splanchnic thrombosis}

Both the ETP and peak ratios were significantly higher in the group with cirrhosis compared to the control group (ETP and peak ratio: $p<0.001$ ); however, no difference was noted for the 'time' thrombin generation marker ratios (lagtime, ttpeak). The same pattern was shown for the thrombin generation markers measured in the presence of thrombomodulin, with significant higher levels for both total and peak thrombin in the patients with cirrhosis compared to controls [ETP: $p=0.006,929$ (2371677) $n M^{*}$ min vs. 593 (296-1406) nM*min. Peak: $p<0.001,191$ (39-250) nM vs. 117 (56-318) nM] (figures 1, 2). 
No significant differences were found when comparing cirrhotic patients with and without PVT.

The ratios for ETP and peak showed weak associations with the CP class (multiple regression analysis, respectively, $p=0.148 p=0.137$ ).

ETP was significantly associated to CP class $(p=0.015)$, whereas peak exhibited a weaker association $(p=0.094)$. ETP in the patients with CP class $C$ was significantly higher than in class A patients [p=0.039, $1513(1349-1677) n^{*}$ min vs. 903 (2371518) $\left.\mathrm{nM}^{*} \mathrm{~min}\right]$, and slightly, but not significantly, higher than ETP in the patients with CP class $B\left[p=0.079,1187(643-1409) n M^{*} \min \right]$.

\section{Thrombin generation and factor VIII in patients with PVT not treated with warfarin compared to patients with cirrhosis}

Both the ETP and peak were significantly higher in the group with cirrhosis than the group with PVT [ETP: $p=0.007,929$ (237-1677) nMmin vs. 636 (275-1689) nMmin. Peak: $p=0.007,191(39-250) n M$ vs. 103 (35-257) nM] (figures 1, 2). The ratios for lagtime, ETP and peak were similarly higher in the group with cirrhosis (lagtime ratio: $p=0.017$, ETP ratio: $p=0.031$, peak ratio: $p=0.020$ ).

When separate analyses were performed (for the groups of patients with C-PVT and NC-PVT) the differences were retained when the cirrhosis group was compared against the group of NC-PVT, with higher ETP, peak, ETP ratio and peak ratio for the patients with cirrhosis [ETP: $p=0.001,929$ (237-1677) nM*min vs. 432 (275-1689) 
$n M^{*}$ min. Peak: $p=0.006,191(39-250) n M$ vs. 79 (35-257). ETP and peak ratio:

$p<0.001$. FVIII: $p=0.006,2.14(1.09-3.86) \mathrm{IU} / \mathrm{mL}$ vs. $1.68(0.86-3.88) \mathrm{IU} / \mathrm{mL}$ ] (figures

$1,2)$. When the results from the cirrhosis group were compared with the group with

C-PVT, no differences were observed.

\section{Thrombin generation and thrombomodulin resistance for patients with PVT,} C-PVT, NC-PVT, Budd-Chiari syndrome, cirrhosis and controls treated with warfarin

No differences were observed between the TG markers in the subgroup of patients with PVT, C-PVT, NC-PVT and BCS treated with warfarin and their respective controls with AF.

\section{Discussion}

We studied patients with three distinct hepatic pathologies - PVT, BCS and cirrhosis with regard to hypercoagulability as reflected by thrombin generation. We used thrombin generation markers as direct markers for hypercoagulability and ratios for the thrombin generation markers in the presence and absence of thrombomodulin. These ratios denote the efficiency of thrombomodulin in the activation of PC and were taken as indexes of hypercoagulability as well as an expression of thrombomodulin resistance, i.e. the greater the ratios the higher the hypercoagulability and the thrombomodulin resistance. 
The main finding was that patients with cirrhosis had higher thrombin generation than controls. The ratios reflecting resistance to the action of thrombomodulin were also higher in the group with cirrhosis. ETP was higher in the groups with CP class B and C compared to CP class A. Our findings confirm the findings by Tripodi et al [24], that hypercoagulability is linked to the presence of CLD.

Seven out of the 47 patients with PVT had hereditary thrombophilia (3 patients with NC-PVT and 2 patients with C-PVT without treatment with warfarin and 2 patients with NC-PVT on warfarin treatment). Five out of 15 patients with BCS (one with without warfarin treatment and 4 on warfarin) also had hereditary thrombophilia. Ten of those patients were heterozygous carriers of the factor $\mathrm{V}$ Leiden, whereas 2 (1 with C-PVT without warfarin and 1 with NC-PVT on warfarin) were carriers of the prothrombin gene mutation. No data were available for the patients with cirrhosis without splanchnic thrombosis or for the controls. The percentage of patients with PVT and factor V Leiden corresponds to the actual prevalence of this mutation in Scandinavia [25]. As this mutation is very common and raises the risk for thrombosis about 3-fold [26], we did not adjust the thrombin generation results for this factor.

Our results show that the resistance to thrombomodulin in patients with PVT is present only when PVT is associated with cirrhosis. Higher total and maximum thrombin concentration and ratios in the group with C-PVT, both in comparison with the control group and the NC-PVT patients, reflects hypercoagulability caused by the underlying cirrhosis, irrespective if PVT is present or not. This finding is in partial contrast with the original hypothesis about the presence of hypercoagulability being linked to the thrombotic incidence. No differences were shown for the "time" 
parameters, i.e. lagtime and ttpeak, indicating that it is mainly the amount of produced thrombin that is important for the hypercoagulability. This could be potentially important when assessing the bleeding risk, for example during invasive procedures, as prolonged lagtime has been associated with increased bleeding risk in patients with factor deficiencies [27]; however, this has not been studied in patients with liver disease.

Thrombin generation in patients with BCS did not differ from that in controls. This is in agreement with the findings from the group with NC-PVT, underscoring the absence of increased thrombin generation in patients with splanchnic venous thrombosis and no cirrhosis. Even if the low number of observations $(n=15)$ in the BCS group limits the conclusions that can be drawn the findings may contribute to a better understanding of this disorder, since large studies in BCS patients are difficult to carry out due to the rarity of the condition [18].

Tripodi et al [7] showed that the median ratio of thrombin generation (with/without thrombomodulin) was significantly higher in cirrhotic patients compared to controls suggesting that patients with CLD might be resistant to the action of thrombomodulin. The hypercoagulability observed in those patients [7] was more pronounced with higher CP class and our study confirms those results; however, due to the low number of patients with $\mathrm{CP}$ class $\mathrm{C}$ for which we were able to obtain thrombin generation profiles $(n=2)$, we refrain from drawing further conclusions from those results. 
We also measured thrombin generation markers and ratios in patients with BCS and PVT on warfarin treatment and compared the results to those from patients on warfarin treatment as primary prophylaxis against stroke. No statistical significant differences were observed, indicating that the haemostatic potential of the patients with BCS and PVT was not affected prior to initiation of warfarin treatment. The hypercoagulability caused by the presence of CLD is balanced by the effect of warfarin. An indirect conclusion could be that patients with liver disease and on warfarin might not have higher bleeding risk than controls (without liver diseases) on warfarin when the PT-INR is within the recommended therapeutic interval.

Recently, Raffa et al [20] reported that ETP in patients with NC-PVT is higher than in healthy controls. The authors used the same assay as we did with the same concentrations of phospholipids and tissue factor, as well as the same final concentration of thrombomodulin $(4 \mathrm{nM})$. This thrombin generation assay does not offer a standardized reference range for normal thrombin generation. Results are therefore compared with controls used in each local laboratory. Even in the absence of a common reference range, thrombin generation assays can be used as a marker for disease. Their blood sampling was at the time of diagnosis, i.e. during the acute phase of the thromboembolic episode. In contrast, the blood sampling for PVT in our study took place at a median of 46 months following diagnosis. This difference in the time could have influenced the differences between our and their results. The finding of hypercoagulability associated with cirrhosis in patients with C-PVT, but not NCPVT, was evident both from ratios and markers in the presence of thrombomodulin and was further confirmed in the cirrhosis group. This reproducibility is one of the 
strengths of our study and implicates cirrhosis as the major driving force behind an observed hypercoagulability.

The thrombin generation markers in our study show a considerable intraindividual variation (tables 2, 3, 4). However it has been reported that thrombin generation exhibits a rather large variation even in the normal population [28] and our results refer to a population with heterogeneous liver disease. As such, it is difficult to interpret the significance of this variation, but it could imply that the results are applicable on large populations, rather than individuals.

It has been reported [29] that addition of phospholipids at concentrations greater than 1.5 micromolar can minimize the influence of residual platelets in the plasma. Since the concentration of phospholipids added to our samples was 4 micromolar, we were able to centrifuge for a second time following thawing of the samples without risking high activation of any residual platelets in the PPP. Additionally, Loeffer et al [30] recently showed that when thrombin generation is measured at the presence of $5 \mathrm{pM}$ TF (as in our study) the effect on the results is negligible even following incubation for varying incubation periods at $4{ }^{\circ} \mathrm{C}$, room temperature and $37^{\circ} \mathrm{C}$, demonstrating the stability of plasma under those conditions.

\section{Conclusions}

We show that there are distinct differences in the haemostatic potential between CPVT and NC-PVT, with C-PVT patients exhibiting a clearly more active coagulation 
activity. Our results could lead to considering longer or more intensive treatment with anticoagulants for C-PVT patients. Higher thrombin generation has been studied as a predictive marker for recurrence in patients with VTE [31], however no prospective studies on the relationship between higher thrombin generation and risk for first time/recurrent PVT have been performed. Such studies are highly warranted as recent data suggest that prophylaxis with low molecular weight heparin (enoxaparin) not only prevents PVT in cirrhotic patients, but also delays the occurrence of hepatic decompensation and improves survival [32]. The small number of patients in each group as well as the wide range in the values of the thrombin generation markers necessitate larger studies.

Acknowledgments: We thank Drs Mårten Werner, Per Sangfelt and Sven Wallerstedt who included patients at their respective hospitals, and laboratory technicians Lena Svensson and Kerstin M. Gustafsson for excellent assistance.

\section{References}

[1] Trotter JF. Coagulation abnormalities in patients who have liver disease. Clin Liver Dis 2006; 10: 665-78

[2] Tripodi A, Mannucci PM. The coagulopathy of chronic liver disease. NEJM 2011; 365: $147-56$

[3] Caldwell SH, Hoffman M, Lisman T, Macik BG, Northup PG, Reddy KR et al. Coagulation disorders and haemostasis in liver disease: pathophysiology and critical assessment of current management. Hepatology 2006; 44: 1039-46 
[4] Lisman T, Caldwell SH, Burroughs AK, Northup PG, Senzolo M, Stravitz RT et al. Hemostasis and thrombosis in patients with liver disease: the ups and downs. $J$ Hepatol 2010; 53: 362-71

[5] Senzolo M, Cholongitas E, Thalheimer U, Riddell A. Agarwal S, Mallett S et al. Heparin-like effect in liver disease and liver transplantation. Clin Liver Dis 2009; 13: $43-53$

[6] Wada H, Usui M, Sakuragawa N. Hemostatic abnormalities and liver diseases. Semin Thromb Hemost 2008; 34: 772-78

[7] Tripodi A, Primignani M, Chantarangkul V, Dell'Era A, Clerici M, de Franchis R et al. An imbalance of pro- vs anti-coagulation factors in plasma from patients with cirrhosis. Gastroenterology 2009; 137: 2105-11

[8] Boks AL, Brommer EJ, Schalm SW, van Vliet HH. Hemostasis and fibrinolysis in severe liver failure and their relation to hemorrhage. Hepatology 1986; 6: 79-86

[9] Tripodi A, Salerno F, Chantarangkul V, Clerici M, Cazzaniga M, Mannucci PM. Evidence of normal thrombin generation in cirrhosis despite abnormal conventional coagulation tests. Hepatology 2005; 41: 553-58

[10] Tripodi A, Primignani M, Mannucci PM. Abnormalities of hemostasis and bleeding in chronic liver disease: the paradigms challenged. Intern Emerg Med 2010; 5: 7-12

[11] Lippi G, Targher G, Favaloro EJ, Franchini M. Venous thromboembolism in chronic liver disease. Semin Thromb Hemost 2011; 37: 66-76

[12] Søgaard KK, Horváth-Puhó E, Grønbaek H, Jepsen P, Vilstrup H, Sørensen HT. Risk of venous thromboembolism in patients with liver disease: a nationwide population-based case - control study. Am J Gastroenterol 2009; 104: 96-101 
[13] Northup PG, Volk-Bednarz A, Berg CL. Predictors of peripheral venous thromboembolism in cirrhosis. AJG 2003; 98: S97

[14] Ali M, Ananthakrishnan AN, McGinley EL, Saeian K. Deep vein thrombosis and pulmonary embolism in hospitalized patients with cirrhosis: a nationwide analysis. Dig Dis Sci 2011; 56: 2152-59

[15] Hemker HC, Giesen P, Al Dieri R, Regnault V, de Smed E, Wagenvoord R et al. The Calibrated Automated Thrombogram (CAT): a universal routine test for hyper and hypocoagulability. Pathophysiol Haemost Thromb 2002; 32: 249-53

[16] Ferral H, Behrens G, Lopera J. Budd-Chiari syndrome. Am J Roentgenol 2012; 199: 737-45

[17] Shetty S. Ghosh K. Thrombophilic dimension of Budd-Chiari syndrome and portal venous thrombosis--a concise review. Thromb Res 2011; 127: 505-12

[18] Rajani R, Melin T, Björnsson E, Broomé U, Sangfelt P, Danielsson A et al. BuddChiari syndrome in Sweden: epidemiology, clinical characteristics and survival - an 18-year experience Liver Int 2009; 29: 253-59

[19] de Franchis R. Evolving consensus in portal hypertension. Report of the Baveno IV consensus workshop on methodology of diagnosis and therapy in portal hypertension. J Hepatol 2005; 43: 167-76.

[20] Raffa S, Reverter JC, Seijo S, Tassies D, Abraldes JG, Bosch J et al. Hypercoagulability in patients with chronic noncirrhotic portal vein thrombosis. Clin Gastroenterol Hepat 2012; 10: 72-78

[21] Rajani R, Björnsson E, Bergquist A, Danielsson A, Gustavsson A, Grip O et al. The epidemiology and clinical features of portal vein thrombosis: a multicentre study. Aliment Pharmacol Ther 2010; 32: 1154-62 
[22] Ponziani FR, Zocco MA, Garcovich M, D'Aversa F, Roccarina D, Gasbarrini A. What we should know about portal vein thrombosis in cirrhotic patients: A changing perspective. World J Gastroenterol 2012; 18: 5014-20

[23] The Thrombogram Guide. Thrombinoscope $\mathrm{BV}^{\circledR}$, Maastricht, the Netherlands.

[24] Tripodi A, Anstee QM, K. Sogaard KK, Primignani M, Valla DC.

Hypercoagulability in cirrhosis: causes and consequences. J Thromb Haemost 2011;

9: 1713-23

[25] Lindahl TL, Lundahl TH, Nilsson L, Andersson CA. APC-resistance is a risk factor for postoperative thromboembolism in elective hip replacement of the hip or knee - a prospective study. Thromb Haemost 1999;81-18-21

[26] Middeltorp S. Is thrombophilia testing useful? Hematology Am Soc Hematol Educ Program 2011;2011:150-5

[27] Zekavat OR, Haghpanah S, Dahghani J, Afrasiabi A, Peyvandi F, Karimi M. Comparison of thrombin generation assay with conventional coagulation tests in evaluation of bleeding risk in patients with rare bleeding disorders. Clin Appl Thromb Hemost 2013 Feb 6 [Epub ahead of print]

[28] Hemker HC, Giesen P, Al Dieri R, Regnault V, de Smedt E, Wagenvoord R et al. Calibrated Automated Thrombin Generation Measurement in Clotting Plasma. Pathophysiol Haemost Thromb 2003; 33: 4-15

[29] Chantarangkul V, Clerici M, Bressi C, Giesen PL, Tripodi A. Thrombin generation assessed as endogenous thrombin potential in patients with hyper- or hypocoagulability. Haematologica 2003; 88: 547-54

[30] Loeffen R, Kleinegris MC, Loubele ST, Pluijmen PH, Fens D, van Oerle R et al. Preanalytic variables of thrombin generation: towards a standard procedure and 
validation of the method. J Thromb Haemost 2012; 10: 2544-54

[31] Besser M, Baglin C, Luddington R, van Hylckama Vlieg A, Baglin T. High rate of unprovoked recurrent venous thrombosis is associated with high thrombin-generating potential in a prospective cohort study. J Thromb Haemost 2008; 6: 1720-25

[32] Villa E, Cammá C, Marietta M, Luongo M, Critelli R, Colopi S et al. Enoxaparin prevents portal vein thrombosis and liver decompensation in patients with advanced cirrhosis. Gastroenterology 2012; 143: 1253-60 
Tables

\begin{tabular}{|c|c|c|c|c|c|}
\hline & PVT & C-PVT & NC-PVT & BCS & Cirrhosis \\
\hline $\mathrm{n}$ & 47 & 11 & 36 & 15 & 24 \\
\hline Male/female & $27 / 20$ & $8 / 3$ & $19 / 17$ & $7 / 8$ & $15 / 9$ \\
\hline Age & $56(24-80)$ & $60(35-74)$ & $56(24-80)$ & $31(24-57)$ & $57(28-73)$ \\
\hline Warfarin treatment & 15 & 2 & 13 & 10 & 0 \\
\hline JAK-2 ${ }^{1}$ & 5 & 0 & 5 & 9 & $x$ \\
\hline Thrombophilia $^{2}$ & 7 & 2 & 5 & 5 & $x$ \\
\hline Hemoglobin (g/L) & $142(113-166)$ & $132(113-166)$ & $142(113-164)$ & $137(117-173)$ & $124(55-163)$ \\
\hline Platelets $\left(\times 10^{9} / \mathrm{L}\right)$ & $219(62-545)$ & 134 (62-352) & $232(79-545)$ & 294 (99-794) & $147(51-391)$ \\
\hline $\mathrm{ALT}^{3}$ (ukat/L) & $0.52(0.13-1.41)$ & $0.55(0.36-1.41)$ & $1.1(0.1-1.2)$ & $0.66(0.19-1.22)$ & $0.68(0.2-2.33)$ \\
\hline Albumin $(\mathrm{g} / \mathrm{L})$ & $39(24-46)$ & $37(24-46)$ & $39(33-46)$ & $42(37-45)$ & $32(22-43)$ \\
\hline Bilirubin (g/L) & $11(5-52)$ & $15(6-52)$ & $10(5-40)$ & $14(8-48)$ & $16(6-265)$ \\
\hline PT-INR ${ }^{4}$ & $1.1(0.9-2)$ & $1.2(1-1.4)$ & $1(0.9-2)$ & $1(1-1.2)$ & $1.2(1-1.9)$ \\
\hline $\mathrm{hsCRP}(\mathrm{mg} / \mathrm{L})$ & $2.45(0.07-39.6)$ & $2.63(0.3-39.6)$ & $2(0.07-31)$ & $1.94(0.12-10.8)$ & $5.4(0.24-24.44)$ \\
\hline FVIII (IU/mL) & $1.82(0.86-3.88)$ & $1.84(0.86-2.84)$ & $1.68(0.86-3.88)$ & $1.54(1-2.28)$ & $2.14(1.09-3.86)$ \\
\hline Antithrombin $(\mathrm{IU} / \mathrm{mL})$ & $0.96(0.69-1.15)$ & $0.92(0-72-1.14)$ & $0.97(0.63-1.15)$ & $1.04(0.86-1.33)$ & $x$ \\
\hline CP-class A/B/C (n) & $\mathrm{N} / \mathrm{A}$ & $6 / 3 / 2$ & $\mathrm{~N} / \mathrm{A}$ & $\mathrm{N} / \mathrm{A}$ & $16 / 6 / 2$ \\
\hline
\end{tabular}

Table 1. Basic characteristics of the patients and controls included in the study expressed as median values (range).

PVT: portal vein thrombosis, C-PVT: cirrhotic portal vein thrombosis, NC-PVT: non cirrhotic portal vein thrombosis, BCS: Budd-Chiari syndrome

1 : patients with Janus kinase-2 mutation

2 : presence of factor $\mathrm{V}$ Leiden and/or prothrombin G20201A mutation 
3: Alanine transaminase

4 : For patients without treatment with warfarin

x: Data not available

N/A: Not applicable

\begin{tabular}{|l|l|l|l|l|l|l|}
\cline { 2 - 7 } \multicolumn{1}{c|}{} & PVT & C-PVT & NC-PVT & BCS & Cirrhosis & Controls \\
\hline $\mathrm{n}$ & 32 & 9 & 23 & 5 & 24 & 21 \\
\hline Lagtime(min)+TM* & $3.2(1.5-28)$ & $2.8(1.5-4.3)$ & $3.3(1.8-28)$ & $3.6(3-4)$ & $3.3(2.7-15.2)$ & $3.5(2.3-5.3)$ \\
\hline ETP(nM*min)+TM & $636(275-1689)$ & $882(406-1274)$ & $432(275-1689)$ & $620(470-1092)$ & $929(237-1677)$ & $593(296-1406)$ \\
\hline Peak(nM)+TM & $103(35-257)$ & $166(102-206)$ & $79(35-257)$ & $102(84-193)$ & $191(39-250)$ & $117(56-318)$ \\
\hline Ttpeak(min)+TM & $6.1(3.8-33)$ & $5.7(3.8-7.2)$ & $6.4(4.2-33)$ & $6.7(5.7-7.2)$ & $6(4.5-18.5)$ & $6(4.7-8.5)$ \\
\hline Lagtime(min)-TM & $3.5(1.5-25)$ & $2.8(1.5-3.8)$ & $3.5(1.8-25)$ & $3.6(3-4.5)$ & $3.2(2.5-7.5)$ & $3.3(2.3-5.3)$ \\
\hline ETP(nM ${ }^{*}$ min)- TM & $1341(388-2609)$ & $991(894-1730)$ & $1425(388-2609)$ & $1263(920-2060)$ & $1352(857-2006)$ & $1585(1108-2244)$ \\
\hline Peak(nM)-TM & $198(33-345)$ & $196(176-244)$ & $79(33-345)$ & $210(172-314)$ & $220(92-307)$ & $280(203-388)$ \\
\hline ttpeak(min)-TM & $6.9(3.5-31)$ & $6.1(3.5-7.8)$ & $7.1(4.2-31)$ & $9.7(7.8-31)$ & $6(4.8-10)$ & $6.3(4.7-8.5)$ \\
\hline Lagtime ratio & $1(0.6-1.4)$ & $1(0.9-1.1)$ & $0.9(0.6-1.4)$ & $1(0.9-1)$ & $1(0.5-3.8)$ & $1(0.9-1.2)$ \\
\hline ETP ratio & $0.5(0.1-1)$ & $0.9(0.3-1)$ & $0.5(0.2-0.9)$ & $0.5(0.4-0.7)$ & $0.7(0.2-1.3)$ & $0.4(0.2-0.8)$ \\
\hline Peak ratio & $0.6(0.1-1.1)$ & $0.9(0.4-1)$ & $0.5(0.1-1.1)$ & $0.5(0.4-0.8)$ & $0.8(0.2-1.3)$ & $0.4(0.2-0.8)$ \\
\hline Ttpeak ratio & $0.8(0.4-2.9)$ & $0.8(0.4-1.1)$ & $0.8(0.6-0.8)$ & $0.6(0.2-0.9)$ & $1(0.6-1.5)$ & $0.4(0.2-0.8)$ \\
\hline
\end{tabular}

Table 2. Thrombin generation markers and ratios [marker measured in the presence(+)/abscence(-) of thrombomodulin] for patients and controls without warfarin treatment.

${ }^{*} \mathrm{TM}$ : thrombomodulin 


\begin{tabular}{|l|l|l|l|l|}
\hline & PVT & C-PVT & NC- PVT & BCS \\
\hline $\mathrm{n}$ & 15 & 2 & 13 & 10 \\
\hline Age & $57(43-68)$ & $58(55-60)$ & $60(43-68)$ & $32(24-56)$ \\
\hline $\mathrm{M} / \mathrm{F}$ & $9 / 6$ & $1 / 1$ & $8 / 5$ & $5 / 5$ \\
\hline PT-INR & $2.4(2-2.8)$ & $2.9(2.5-3.4)$ & $2.5(2.2-3.3)$ & $2.6(1.9-3.5)$ \\
\hline Lagtime(min)+TM & $6.2(4-14)$ & $5.6(4.8-6.3)$ & $6.2(4-14)$ & $5.2(4-12)$ \\
\hline ETP(nMmin)+TM & $347(172-972)$ & $237(161-313)$ & $350(161-972)$ & $344(118-615)$ \\
\hline Peak(nM)+TM & $73(34-171)$ & $47(34-60)$ & $73(34-171)$ & $73(25-170)$ \\
\hline Ttpeak(min)+TM & $8.7(7.2-19.8)$ & $8.3(7.7-9)$ & $8.7(7.2-19.8)$ & $7.8(7-22.8)$ \\
\hline Lagtime(min)-TM & $6(4.1-12)$ & $6.1(6-6.2)$ & $5.5(4.1-12)$ & $4.8(4.3-16)$ \\
\hline ETP(nM*min)-TM & $452(192-1640)$ & $345(300-390)$ & $470(192-1640)$ & $472(246-1476)$ \\
\hline Peak(nM)-TM & $77(32-250)$ & $51(36-66)$ & $84(32-250)$ & $94(42-232)$ \\
\hline Ttpeak(min)-TM & $8.7(6.8-16.3)$ & $9.8(9.3-10.3)$ & $8.7(6.8-16.3)$ & $8(6.2-11)$ \\
\hline Lagtime ratio & $1.04(0.8-1.5)$ & $0.9(0.8-1.06)$ & $1.04(0.8-1.5)$ & $1.1(0.96-1.2)$ \\
\hline ETP ratio & $0.7(0.04-1.04)$ & $0.7(0.5-0.8)$ & $0.7(0.04-1.04)$ & $0.7(0.2-1.07)$ \\
\hline Peak ratio & $0.9(0.04-1.2)$ & $0.9(0.9-0.95)$ & $1(0.04-1.2)$ & $0.9(0.25-1.7)$ \\
\hline Ttpeak ratio & $1(0.45-1.7)$ & $0.9(0.8-1.07)$ & $1(0.45-1.7)$ & $1.1(0.7-1.47)$ \\
\hline
\end{tabular}

Table 3. Thrombin generation markers and ratios [marker measured in the presence(+)/abscence(-) of thrombomodulin] for patients with PVT. C-PVT, NC-PVT and BCS on warfarin treatment. 


\begin{tabular}{|l|l|l|l|l|}
\hline & PVT controls & C-PVT controls & NC- PVT controls & BCS controls \\
\hline $\mathrm{n}$ & 15 & 2 & 13 & 10 \\
\hline Age & $59(40-72)$ & $61(50-71)$ & $62(40-72)$ & $49(35-61)$ \\
\hline $\mathrm{M} / \mathrm{F}$ & $9 / 6$ & $1 / 1$ & $8 / 5$ & $5 / 5$ \\
\hline $\mathrm{PT}-\mathrm{INR}$ & $2.5(2.1-3.3)$ & $2.9(2.5-3.3)$ & $2.5(2.1-3.3)$ & $2.6(2-3.5)$ \\
\hline Lagtime(min)+TM & $6(4-11)$ & $6.2(6.2-6.3)$ & $5.5(4-11)$ & $6.3(3.3-9)$ \\
\hline ETP(nMmin)+TM & $233(103-696)$ & $234(202-267)$ & $233(103-836)$ & $305(202-696)$ \\
\hline Peak(nM)+TM & $99(40-176)$ & $94(88-99)$ & $103(40-176)$ & $93(61-176)$ \\
\hline Ttpeak(min)+TM & $8.3(5.3-14.2)$ & $8.7(8.7-8.8)$ & $8.2(5.3-14.2)$ & $8.9(5.3-11.6)$ \\
\hline Lagtime(min)-TM & $4.5(2.9-16)$ & $5.2(4.5-5.8)$ & $4.3(3.5-16)$ & $5(2.9-8.8)$ \\
\hline ETP(nMmin)-TM & $391(181-1014)$ & $614(391-836)$ & $377(181-1014)$ & $621(331-1266)$ \\
\hline Peak(nM)-TM & $94(22-186)$ & $115(76-153)$ & $94(22-186)$ & $131(46-198)$ \\
\hline Ttpeak(min)-TM & $7.1(5.3-21)$ & $8(7.1-8.8)$ & $6.8(5.3-21)$ & $7.7(5.2-13)$ \\
\hline Lagtime ratio & $0.9(0.6-1.8)$ & $0.8(0.7-0.9)$ & $0.9(0.6-1.8)$ & $1.2(0.8-1.4)$ \\
\hline ETP ratio & $1.2(0.3-1.3)$ & $0.9(0.5-1.2)$ & $0.8(0.3-1.3)$ & $0.7(0.2-0.9)$ \\
\hline Peak ratio & $1.2(0.2-1.6)$ & $1.2(0.8-1.5)$ & $1.2(0.2-1.6)$ & $0.9(0.7-1.6)$ \\
\hline Ttpeak ratio & $0.9(0.7-1.9)$ & $0.9(0.8-1.02)$ & $0.9(0.7-1.9)$ & $1.1(0.7-1.3)$ \\
\hline
\end{tabular}

Table 4. Thrombin generation markers and ratios [marker measured in the presence(+)/abscence(-) of thrombomodulin] for the controls for the patients with PVT, C-PVT, NC-PVT and BCS on warfarin treatment. 


\section{Figures:}

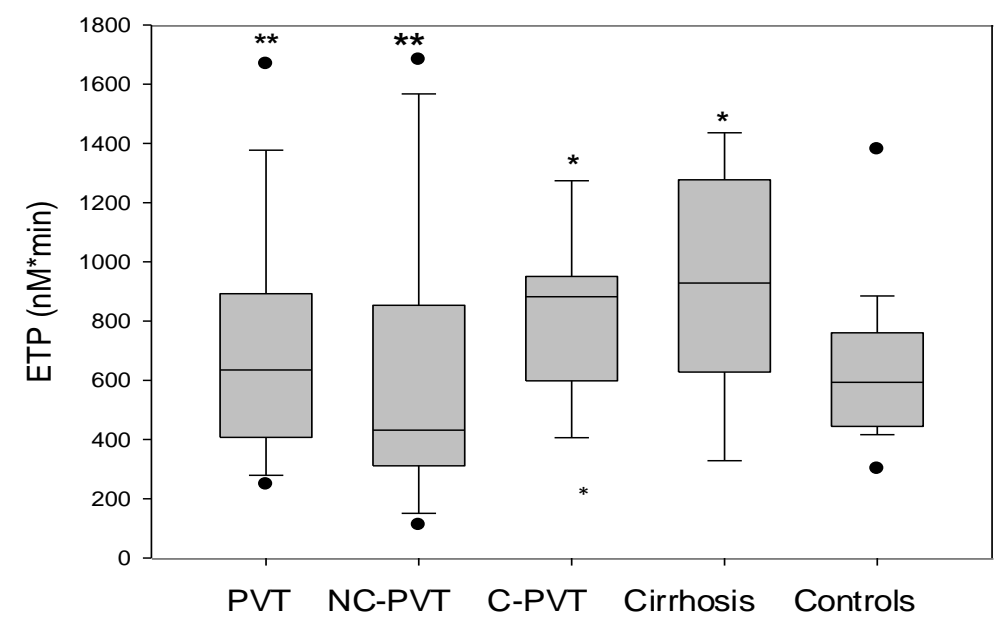

Figure 1. ETP (measured in the presence of thrombomodulin) for the patients with PVT ( $n=32)$, NC-PVT $(n=23)$, C-PVT $(n=9)$, cirrhosis $(n=24)$ and controls $(n=21)$ without warfarin treatment.

* statistically significant higher ETP compared to controls [C-PVT: $p=0.044$, 882 (4061274) $n M^{*} \min$ vs. 593 (296-1406) $n M^{*}$ min and cirrhosis: ETP: $p=0.006$, ETP 929 (237-1677) nM*min vs. $\left.593(296-1406) n^{*} \min \right]$.

${ }^{* *}$ statistically significant lower ETP compared to patients with cirrhosis [PVT: $\mathrm{p}=0.007,929(237-1677) \mathrm{nM}^{*} \min$ vs. $623(65-1689) n M^{*} \min$ and NC-PVT: $\mathrm{p}=0.001$, 929 (237-1677) nM*min vs. 432 (65-1689) nMmin]. 


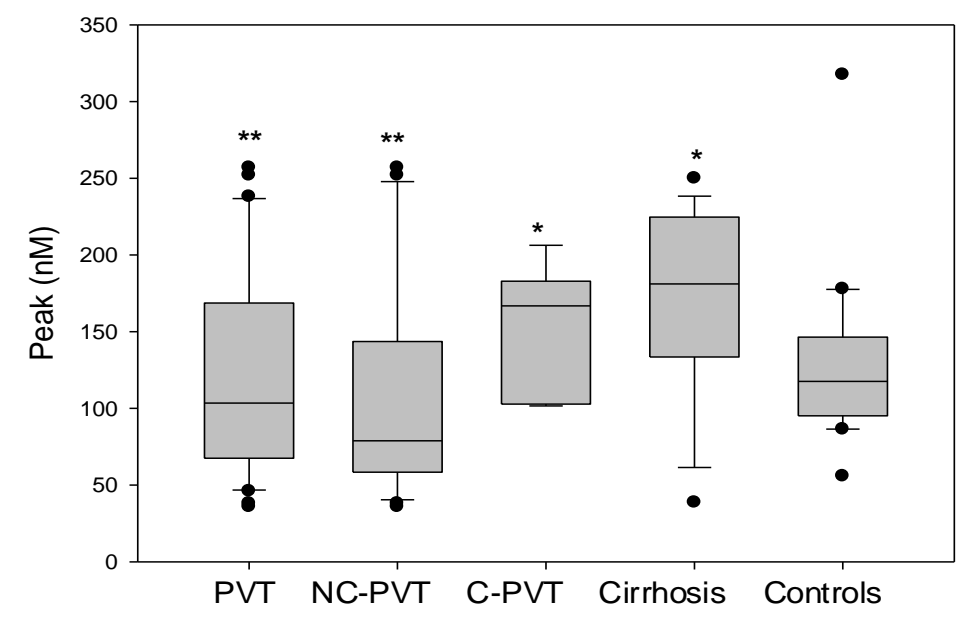

Figure 2. Peak thrombin (measured in the presence of thrombomodulin) for the patients with PVT $(n=32)$, NC-PVT $(n=23)$, C-PVT $(n=9)$, cirrhosis $(n=24)$ and controls $(n=21)$ without warfarin treatment.

* statistically significant higher peak compared to controls [C-PVT: $p=0.020,166$ (102-206) nM vs 117 (56-318) nM and cirrhosis: $p<0.001,191(39-250) n M$ vs. 117 (56-318) nM].

${ }^{* *}$ statistically significant lower peak compared to patients with cirrhosis [PVT: $\mathrm{p}=0.007,191(39-250) \mathrm{nM}$ vs. 103 (35-257) nM and NC-PVT: $p=0.006,191(39-250)$ nM vs. 79 (35-257)]. 